\title{
Canal mandibular accesorio: análisis de su prevalencia y aspecto imagenológico
}

\section{Accessory mandibular canal: analysis of prevalence and imaging appearance}

\author{
Suazo Galdames I*, Zavando Matamala D**, Cantín López M*
}

\section{RESUMEN}

Introducción: La presencia de canales accesorios en la mandíbula ha sido reportada en la literatura, siendo cuestionado el carácter de variación anatómica de su presentación. El reconocimiento de estas formaciones tiene gran importancia en la práctica de la implantología. El propósito de este estudio fue determinar la prevalencia del canal mandibular accesorio y establecer su trayecto y relación con elementos anatómicos circundantes, en mandíbulas secas de individuos adultos pertenecientes al museo de cráneos de la Universidad Federal de São Paulo, mediante imágenes tomografías computarizadas.

Material y método: Se analizaron 294 mandíbulas de sexo y edad conocidos, pertenecientes a 195 hombres y 99 mujeres, de entre 18 y 100 años de edad, las cuales fueron inspeccionadas visualmente y con ayuda de un indicador metálico, el cual se mantuvo para obtener sus imágenes en tomografía axial computarizada, examinándose su proyección panorámica y en cortes coronales.

Resultados: La prevalencia del canal mandibular accesorio fue de 1,7\% (5/294), todos ellos se extendieron paralelos al canal principal, proyectándose anteriormente al foramen mentoniano, con una corticalización parcial.

Discusiones: El canal mandibular accesorio presenta una baja prevalencia en la muestra analizada, proponemos que su presencia está asociada a una proyección independiente de los ramos incisivos del nervio alveolar inferior, cuya evaluación es necesaria para evitar su compromiso en los procedimientos implantológicos mandibulares.

Palabras clave: Canal mandibular, mandíbula, nervio alveolar inferior, implantología.

\section{SUMMARY}

Introduction: The presence of accessory canals in the mandible has been reported in the literature, still challenge the view that anatomical variation of submission. The recognition of these formations is of great importance in implantology practice. The purpose of this study was to determine the prevalence of accessory mandibular canal and set their course and relationship to surrounding anatomic elements in dry mandibles of adult skulls belonging to the Museum of the Universidade Federal de São Paulo, using computed tomography images.

Matherial \& Method: 294 known sex and age jaws were analyzed, belonging to 195 men and 99 women, aged between 18 and 100 years old, which were inspected visually and using a metal indicator, which is held to obtain their images in computed axial tomography, examining its panoramic view and coronal sections.

Results: The prevalence of accessory mandibular canal was $1.7 \%$ (5/294), all extended parallel to the main canal, projecting above the mental foramen, with a partial corticalization.

* Morfología. Universidad de Talca, Chile.

** Departamento de Morfología y Genética. Universidad Federal de São Paulo. Brasil. 
Discussion: The accessory mandibular canal has a low prevalence in the sample, we propose that their presence is associated with an independent projection of the branches of the inferior alveolar nerve, whose evaluation is necessary to avoid its commitment in mandibular implant procedures.

Key words: Mandibular canal, jaw, alveolar inferior nerve, implantology.

Fecha de recepción: 6 de octubre 2009.

Aceptado para publicación: 13 de octubre 2009.

Suazo Galdames I, Zavando Matamala D, Cantín López M. Canal mandibular accesorio: análisis de su prevalencia y aspecto imagenológico. Av. Odontoestomatol 2011; 27 (2): 85-90.

\section{INTRODUCCIÓN}

El canal mandibular es una formación que se inicia en la cara medial de la rama de la mandíbula a partir del foramen mandibular y se continúa en dirección inferolateral, relacionándose a su paso con las raíces de los molares y premolares inferiores, corticalizado en la mayor parte de su trayecto para terminar en el foramen mental $(1,2)$. Al ser examinado transversalmente, puede mostrar una forma ovalada, circular o piriforme en cuyo interior se encuentra el paquete neurovascular alveolar inferior que está constituido por vasos sanguíneos y el nervio alveolar inferior, ramo de la tercera división del nervio trigémino (3).

Debido a la posición y curso del canal mandibular, el análisis anatómico realizado a través de los exámenes imagenológicos, permiten tanto su propia evaluación como las estructuras circundantes, detección de procesos patológicos, estimación de la cantidad, altura y calidad del hueso. Estos datos son considerados fundamentales para la planificación y ejecución de procedimientos quirúrgicos implantológicos que involucren a la mandíbula (4).

Generalmente este canal se presenta de manera única, sin embargo, la presencia de un segundo canal o tercer canal mandibular de tipo accesorio ha sido reportado en la literatura $(3,5-16)$.

La presencia y formación de un canal mandibular accesorio se explica durante el desarrollo embrionario, alrededor de la 7 semana de desarrollo, el nervio alveolar inferior se presenta como tres ramos nerviosos separados, los que se dirigen a inervar los 3 grupos de dientes mandibulares. Primero aparece un ramo para las piezas anteroinferiores, seguido por uno para los molares temporales y finalmente uno para los molares permanentes; estos ramos nerviosos se van fusionando durante el crecimiento prenatal mandibular formando un nervio alveolar inferior único, el cual es rodeado por tejido óseo membranoso conformando el canal mandibular, sin embargo, al ocurrir una fusión incompleta de alguno de estos ramos nerviosos, el tejido se osificará alrededor de él dando origen a un canal mandibular accesorio (17).

La prevalencia en que se presenta el canal mandibular accesorio se ha descrito de manera variada. Grover $\mathcal{E}$ Lorton (18) lo han reportado en un 0,08\%, Sanchís y cols. (8) en un $0,35 \%$, Zografos y cols. (19) en un $0,4 \%$, Nortjé y cols. (20) en un $0,9 \%$, Langlais y cols. (21) en un $0,95 \%$ y Durst $\varepsilon$ Snow (22) en un $8,3 \%$ de los casos, todos ellos a través de radiografías panorámicas. Naitoh y cols. (15) lo reportó en el $7 \%$ mediante el análisis de imágenes tomográficas computarizadas.

La presencia de un canal mandibular accesorio puede afectar de manera decisiva la selección de un sitio a ser tratado con implantes (15), debido al riesgo de lesión del paquete neurovascular alveolar inferior, causando discapacidad sensorial postoperatoria. Por tanto, el conocimiento de la existencia, posición, curso de un canal mandibular accesorio y el grosor de sus corticales es imprescindible para 
realizar un correcto diagnóstico y evaluación de la zona mandibular (23), permitiendo la adecuada selección y ubicación de los implantes dentales, a fin de prevenir las complicaciones postquirúrgicas.

El propósito de este estudio fue analizar la prevalencia del canal mandibular accesorio en mandíbulas humanas maceradas y determinar su trayecto y relaciones con elementos anatómicos circundantes a través de tomografía axial computarizada.

\section{MATERIAL Y MÉTODO}

En este estudio se utilizaron 294 mandíbulas secas de individuos adultos brasileños, con registro de sexo y edad, obtenidas mediante un muestreo no probabilístico por conveniencia a partir de la colección de la Universidad Federal de São Paulo. En la tabla 1 encontramos las características de la muestra analizada de acuerdo a sexo y edad.

Las mandíbulas fueron inspeccionadas visualmente y con la ayuda de un indicador metálico flexible de $0,1 \mathrm{~mm}$ de diámetro en busca del canal mandibular accesorio.

Se consideró presente el canal mandibular accesorio cuando se observó:

1. Un foramen en la cara medial de la rama mandibular, próximo, pero independiente del foramen del canal mandibular, como lo indica la figura 1.

2. Que el foramen descrito en el punto precedente origina un canal que puede ser recorrido desli-

\begin{tabular}{|c|c|c|c|c|c|}
\hline \multicolumn{6}{|c|}{$\begin{array}{l}\text { TABLA 1.- CARACTERÍSTICAS DE } \\
\text { LA MUESTRA DE } 294 \text { MANDÍBULAS } \\
\text { DE LA COLECCIÓN DE LA UNIFESP } \\
\text { DE ACUERDO A SEXO Y EDAD }\end{array}$} \\
\hline & \multirow{2}{*}{$\mathrm{n}$} & \multicolumn{3}{|c|}{ Edad } & \multirow{2}{*}{ SD } \\
\hline & & Mínimo & Máximo & Media & \\
\hline Hombre & 195 & 20 & 82 & 43,95 & 13.741 \\
\hline Mujer & 99 & 18 & 100 & 39,15 & 16.892 \\
\hline
\end{tabular}

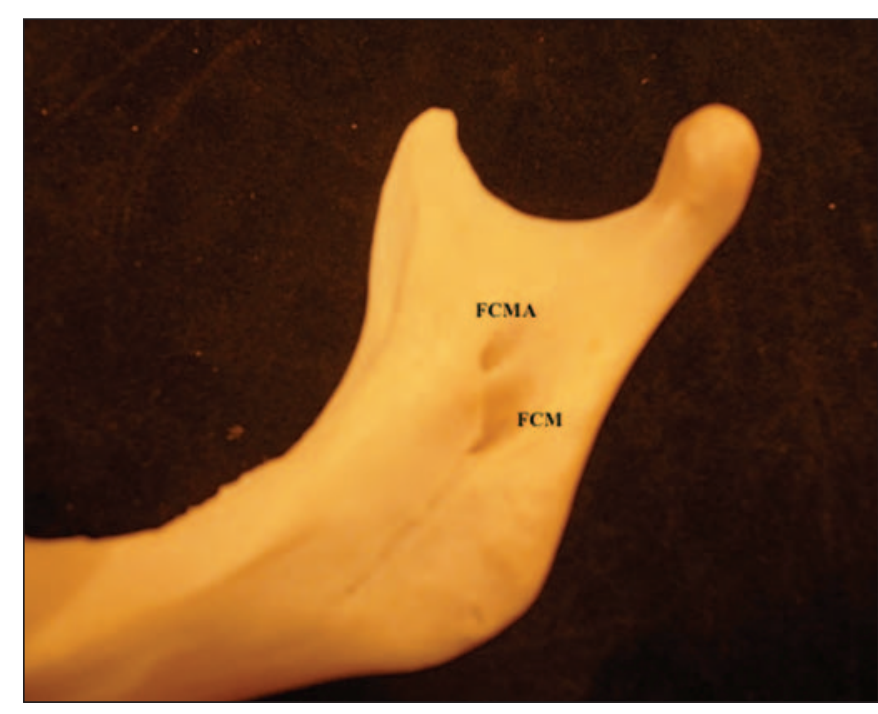

Fig. 1. Vista medial de la rama mandibular de un individuo de 64 años de sexo femenino. FCMA: Foramen del canal mandibular accesorio. FCM: Foramen del canal mandibular.

zando un indicador metálico. Esto se verificó mediante la obtención de una imagen de Tomografía Axial Computarizada, utilizando un equipo NewTom 9000, como se observa en la figura 2, a partir de la cual se describió el trayecto del canal mandibular accesorio y las características de sus corticales.

Con los datos obtenidos se calculó la prevalencia del canal mandibular accesorio en la muestra analizada.

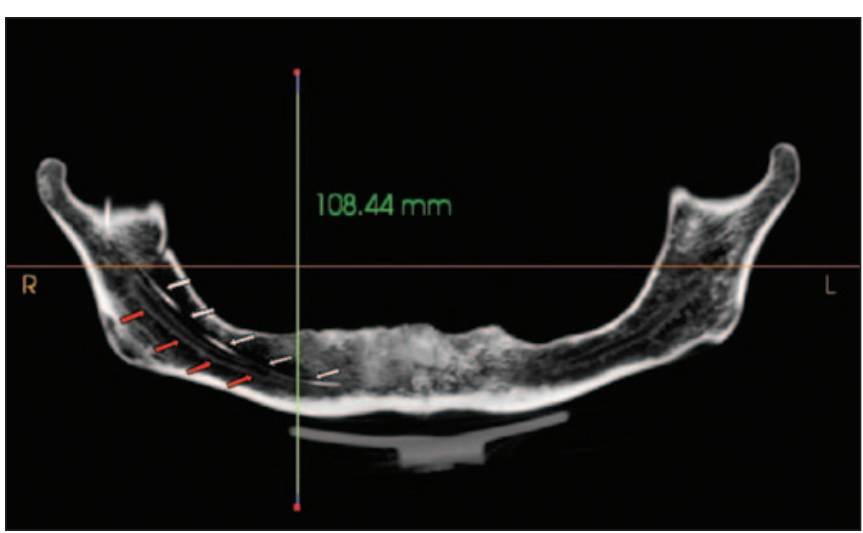

Fig. 2. Imagen panorámica de Tomografía axial computarizada de mandíbula de individuo de 64 años de sexo femenino. Las flechas blancas indican el trayecto del indicador en el canal mandibular accesorio, las flechas rojas indican la cortical del canal mandibular. 


\section{RESULTADOS}

La prevalencia del canal mandibular accesorio fue de 1,7\% (5/294), en todos los casos se encontró de manera unilateral. La distribución por lado analizado se observa en la tabla 2 .

De acuerdo a las características morfológicas, en los 5 casos que fue encontrado un canal mandibular accesorio, este siguió un trayecto descendente hacia anterior, describiendo una curva de concavidad superior. Todos ellos se extendieron paralelos al canal mandibular principal, proyectándose anteriormente hacia el foramen mentoniano, con una corticalización parcial, esto se observa en la figura 3.

\section{DISCUSIÓN}

En este estudio encontramos una prevalencia de $1,7 \%$ de canales mandibulares accesorios identificados a partir del uso de tomografía axial computarizada, lo cual representa una baja frecuencia de esta variación anatómica. Este dato es ligeramente superior a lo reportado por Grover $\mathcal{E}$ Lorton (18), Sanchís y cols. (8), Zografos y colaboradores (19), Nortjé y cols. (20) y Langlais y cols. (21), lo cual puede ser explicado por el tipo de imagen utilizada por estos autores que correspondió a ortopantomografías, la cual presenta una menor resolución en comparación con las imágenes obtenidas por el uso de tomógrafos computarizados. Al momento de comparar la prevalencia obtenida en nuestro estudio con otro donde se usó también tomografías computarizadas vemos que nuestro $1,7 \%$ es bastante inferior al $7 \%$ reportado por Naitoh y cols. (15).

\begin{tabular}{|c|c|c|c|}
\hline \multicolumn{4}{|c|}{$\begin{array}{l}\text { TABLA 2.- DISTRIBUCIÓN POR LADO } \\
\text { DE PRESENTACIÓN DEL CANAL } \\
\text { MANDIBULAR ACCESORIO }\end{array}$} \\
\hline & Frecuencia & Hombre & Mujer \\
\hline Ausente.... & 289 & 192 & 97 \\
\hline Lado derecho ............ & 3 & 2 & 1 \\
\hline Lado izquierdo ........... & 2 & 1 & 1 \\
\hline Total ............................... & 294 & 195 & 99 \\
\hline
\end{tabular}

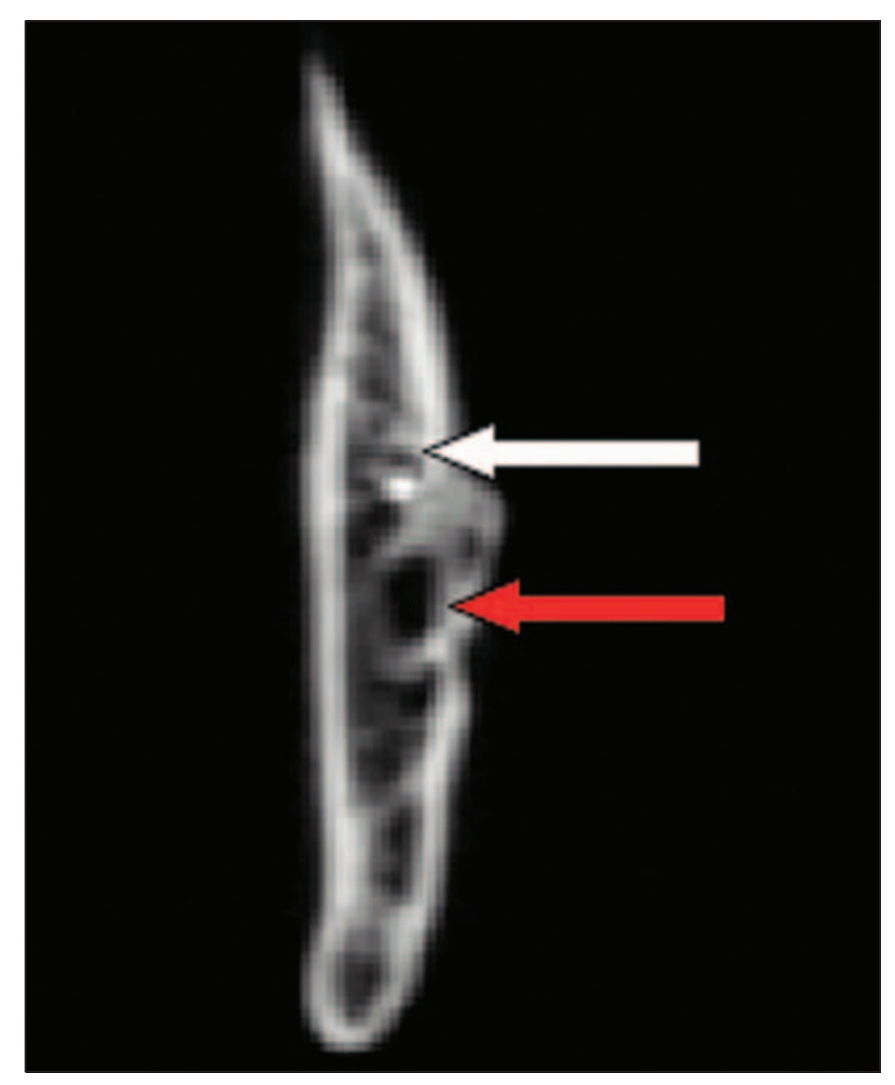

Fig. 3. Imagen coronal de Tomografía computarizada de mandíbula, la flecha blanca indica el canal mandibular accesorio (nótese su corticalización parcial) y la flecha roja muestra el canal mandibular principal.

En relación al trayecto descrito por los canales mandibulares accesorios identificados en nuestra muestra, todos ellos se extendieron paralelos al canal mandibular principal, proyectándose anteriormente hacia el foramen mental, con una corticalización parcial. Ninguno de ellos presentó duplicación manteniéndose un patrón unitario hasta el final de su recorrido.

Al respecto en la literatura, se ha descrito que no existe un patrón específico de división o duplicación de los canales mandibulares accesorios, de haberlos dobles o triples, éstos se encuentran a nivel del cuerpo mandibular alrededor de un canal principal y posiblemente pueden terminar en un foramen mental secundario (24).

La presencia de variaciones anatómicas del canal mandibular ya sea en número como distribución, toma especial importancia al momento de planificar intervenciones quirúrgicas del área, por el riesgo que 
significan las complicaciones nerviosas asociadas al daño del nervio alveolar inferior, siendo una de las complicaciones más graves en la cirugía de implantes mandibulares, la que suele tener un difícil manejo.

Implicación clínica en presencia de un canal mandibular accesorio es el fracaso del bloqueo anestésico realizado mediante la técnica convencional para el nervio alveolar inferior $(8,13)$, haciendo difícil predecir su eficacia. Se ha reportado que en casos de canales accesorios bífidos, la anestesia alta del nervio alveolar inferior utilizando técnicas alternativas como Gow-Gates y Akinosi (en casos de pacientes con limitación de apertura oral) (24). En ambas técnicas puede haber riesgo de punción de estructuras como la arteria maxilar y el plexo pterigoideo (25). Estas técnicas deben ser utilizadas cuando el examen imagenológico evidencia canales mandibulares accesorios y cuando la anestesia al nervio alveolar inferior convencional no es suficiente.

Además, posibles complicaciones durante la cirugía del tercer molar y en casos de trauma donde las fracturas de la mandíbula deben manejarse con cuidado al momento de su reducción para que el paquete neurovascular quede alineado, se hace más difícil en presencia de un segundo paquete neurovascular situado en un plano diferente. En otros procedimientos quirúrgicos tales como la osteotomía mandibular aumenta la complejidad con la adición de un segundo paquete neurovascular accesorio (3).

Con la edad y la pérdida de piezas dentarias, la mandíbula se reduce en altura debido a la reabsorción del tejido óseo, haciendo que el canal mandibular y el foramen mental se encuentren más cerca del reborde alveolar, siendo aún más crítico en mandíbulas completamente desdentadas, donde la reabsorción del proceso alveolar es más amplia y progresiva (26), haciendo que el alto riesgo de daño del canal mandibular en procedimientos de inserción de implantes sea aún mayor si existe algún canal accesorio. La lesión del contenido del canal mandibular afecta tanto a mandíbulas desdentadas parciales como totales con atrofia severa del hueso mandibular, donde el nervio alveolar inferior se conserva manteniendo sus fibras con la capacidad de proporcionar respuesta a estímulos de dolor, temperatura, tacto, presión y propiocepción al igual que en mandíbulas dentadas (27).
En vista de todas estas consideraciones clínicas, se sugiere que el estudio imagenológico de la zona que contiene al canal mandibular sea realizado usando de tomografías axiales de manera preferente a la ortopantomografía, en relación a esto Naitoh y colaboradores (15) ya nos señala que la detección de un canal mandibular accesorio, mediante tomografías podría reducir las tasas de lesión nerviosa y hemorragia consecuencia de la cirugía implantológica, justificando el uso de tomografía como el examen de elección en esta área.

No obstante, la baja prevalencia de esta variación anatómica, es de especial importancia conocerla para poder considerarla una posibilidad en nuestros pacientes.

\section{BIBLIOGRAFÍA}

1. Kaufman E, Serman NJ, Wang PD. Bilateral mandibular accessory foramina and canals: a case report and review of the literature. Dentomaxillofac Radiol 2000;29:170-5.

2. Suazo GIC, Morales HCA, Cantín LMG, Zavando MDA. Aspectos biométricos del canal mandibular. Int J Morphol 2007;25:811-6.

3. Claeys V, Wackens G. Bifid mandibular canal: literature review and case report. Dentomaxillofac Radiol 2005;34:55-8.

4. Tsuji Y, Muto T, Kawakami J, Takeda S. Computed tomographic analysis of the position and course of the mandibular canal: relevance to the sagittal split ramus osteotomy. Int J Oral Maxillofac Surg 2005;34:243-6.

5. Driscoll CF. Bifid mandibular canal. Oral Surg Oral Med Oral Pathol 1990;70:807.

6. Rouviére H, Delmas A. Anatomía Humana Descriptiva, Topográfica y Funcional. 10a. ed. Barcelona: Masson, 1999.

7. Figún M, Garino R. Anatomía Odontológica Funcional y Aplicada. 2a. Ed. Buenos Aires: El Ateneo, 2001. 
8. Sanchís JM, Peñarrocha M, Soler F. Bifid mandibular canal. J Oral Maxillofac Surg 2003;61: 422-4.

9. Latarjet M, Ruiz-Liard A. Atlas de Anatomía. 2a. ed. Buenos Aires: Panamericana, 2004.

10. Auluck A, Pai KM, Mupparapu M. Multiple mandibular nerve canals: radiographic observations and clinical relevance. Report of 6 cases. Quintessence Int 2007;38(9):781-7.

11. Auluck A, Pai KM. Trifid mandibular nerve canal. Dentomaxillofac Radiol 2005;34(4):259.

12. Rouas P, Nancy J, Bar D. Identification of double mandibular canals: literature review and three case reports with CT scans and cone beam CT. Dentomaxillofac Radiol 2007;36:34-8.

13. Wadhwani P, Mathur RM, Kohli M, Sahu R. Mandibular canal variant: a case report. J Oral Pathol Med 2008;37:122-4.

14. Naitoh M, Hiraiwa Y, Aimiya H, Gotoh M, Ariji Y, Izumi M, Kurita K, Ariji E. Bifid mandibular canal in Japanese. Implant Dent 2007;16:24-32.

15. Naitoh M, Hiraiwa Y, Aimiya H, Ariji E. Observation of bifid mandibular canal using cone-beam computerized tomography. Int J Oral Maxillofac Implants 2009;24:155-9.

16. Miloglu O, Yilmaz AB, Caglayan F. Bilateral bifid mandibular canal: a case report. Med Oral Patol Oral Cir Bucal 2009;14:E244-6.

17. Chávez-Lomeli ME, Mansilla Lory J, Pompa JA, Kjaer I. The human mandibular canal arises from three separate canals innervating different tooth groups. J Dent Res 1996;75:1540-4.

18. Grover PS, Lorton L. Bifid mandibular nerve as a possible cause of inadequate anesthesia in the mandible. J Oral Maxillofac Surg 1983;41:177-9.

19. Zografos J, Kolokoudias M, Papadakis E. The types of mandibular canal. Hell Period Stomat Gnathopathoprosopike Cheir 1990;5:17-20.
20. Nortjé CJ, Farman AG, Grotepass FW. Variations in the normal anatomy of the inferior dental (mandibular) canal: a retrospective study of panoramic radiographs from 3612 routine dental patients. Br J Oral Surg 1977;15:55-63.

21. Langlais RP, Broadus R, Glass BJ. Bifid mandibular canals in panoramic radiographs. J Am Dent Assoc 1985;110:923-6.

22. Durst JH, Snow JE. Multiple mandibular canals: oddities or fairly common anomalies? Oral Surg Oral Med Oral Pathol 1980;49:272-3.

23. Angelopoulos $C$, Thomas SL, Hechler S, Parissis $N$, Hlavacek M. Comparison between digital panoramic radiography and cone-beam computed tomography for the identification of the mandibular canal as part of presurgical dental implant assessment. J Oral Maxillofac Surg 2008;66:2130-5.

24. DeSantis JL, Liebow C. Four common mandibular nerve anomalies that lead to local anesthesia failures. J Am Dent Assoc 1996;127:1081-6.

25. Suazo Galdames IC, Cantín López MG, Zavando Matamala DA. Inferior alveolar nerve block anesthesia via the retromolar triangle, an alternative for patients with blood dyscrasias. Med Oral Patol Oral Cir Bucal 2008;13:E43-7.

26. Polland KE, Munro S, Reford G, Lockhart A, Logan G, Brocklebank L, McDonald SW. The mandibular canal of the edentulous jaw. Clin Anat 2001;14:445-52.

27. Wadu SG, Penhall B, Townsend GC. Morphological variability of the human inferior alveolar nerve. Clin Anat 1997;10:82-7.

\section{CORRESPONDENCIA}

Prof. Dr. Iván Suazo Galdames

Morfología

Avenida Lircay, s/n, oficina $\mathrm{n}^{\circ} 104$

Universidad de Talca, Chile

E-mail: isuazo@utalca.cl 